\title{
L'orientalisme et le goût pour le pays des pharaons
}

Amira Ahmed Moustafy Ibrahim

Doctorante au département de français

Faculté Al-Alsun

Université de Minia

a_alsun@yahoo.com

\begin{abstract}
Le mouvement d'orientalisme a été né grâce à l'expédition française en Égypte qui a donné lieu depuis deux siècles à d'innombrables récits, études, œuvres littéraires et commentaires. En effet, elle a enflammé littéralement l'imaginaire des Occidentaux. Dans l'esprit de Bonaparte, il s'agissait d'une triple conquête: la conquête militaire, d'abord, pour soustraire aux Anglais un pays riche et bien placé sur la route des Indes, la conquête politique, ensuite, pour rallier les égyptiens et leur apporter les Lumières de la civilisation occidentale et la conquête scientifique, enfin, pour étudier un pays fascinant, grâce à quelque 160 savants et artistes accompagnant 50000 soldats et marins. L'expédition a permis de réveiller l'Egypte et de l'introduire dans la modernité et dès lors, le goût oriental a envahi la littérature française. La plupart des écrivains et des artistes étaient bien instruits et ont séjourné un certain de temps dans les pays d'Orient. Il est normal que le début du XVIII siècle vienne continuer dans la même tendance orientale. Mais après la traduction des Milles et une nuit de Galland, la France n'a pas cessé de contempler cet Orient pur, riche et imaginaire. Un grand nombre d'écrivains se dirigeaient vers cette fiction orientale. L'Orient est devenu le sujet littéraire dominant et le plus demandé
\end{abstract}

\section{Mots clés}

L'autre - le voyage - les écrivains voyageurs - l'Expédition de Bonaparte - l'intérêt pour l'Égypte.

\section{Introduction}

L'orientalisme est un terme répandu depuis l'année 1830 qui ne désigne pas seulement un style, mais plutôt un climat. Il a paru au XVII ${ }^{\text {ème }}$ siècle et s'est développé dans la peinture française aux XVIII ${ }^{\text {ème }}$ et XIX ${ }^{\text {ème }}$ siècles. Le mouvement de l'orientalisme a commencé avec la mode des Turqueries. Mais, il a connu au 
XIX $^{\text {ème }}$ siècle une évolution très importante: Victor Hugo a noté en 1829, dans la préface des Orientales, que l'Orient est devenu une préoccupation générale, pour cela, il est juste de dire que l'orientalisme, c'est l'Orient vu par les yeux de son opposé, l'Occident; c'est le regard qu'il (Occidental) porte sur l'Autre. Le terme d'orientalisme a donné lieu à des représentations parfois imaginaires d'un Orient tout droit sorti des Mille et Une Nuits. Ces représentations étaient pleines des tableaux descriptifs des palais, du Harem et des momies.

\section{Le voyage et l'intérêt pour l'Antiquité égyptienne}

La France s'est intéressée depuis des siècles longs à l'Orient. D'ailleurs, cet intérêt est très ancien, et remonte à l'époque des Croisades. Le goût de l'Orient lointain était au début attaché aux intentions religieuses (à travers les missionnaires de l'église), à fin de chercher cette religion qui menace le christianisme. Puis le mouvement a acquiert une certaine empreinte coloniale, à travers les campagnes militaires, comme celle de Bonaparte sur l'Égypte en 1798. Le motif colonial s'est enrichi pour mettre la main sur les plus riches colonies du nord de l'Afrique et ouvrir de nouveaux marchés commerciaux. Ce goût est devenu plus fort vers la fin du XVIII ${ }^{\text {ème }}$ siècle avec la faiblesse de l'empire ottoman. Les Européens ont voyagé pour découvrir l'autre côté du monde: l'Orient et, ils savent bien que cet Orient est la source des civilisations et ses cultures les fascinaient:

«Depuis l'expédition de l'Égypte par Napoléon Bonaparte jusqu'à nos jours le regard - ou les regards- arabe est resté tributaire des différentes conjonctures historiques que traverse le monde arabe.» (Affaya 1999: 217) Pour l'homme de Lettres, le voyage dans le temps équivaut à un retour aux sources. La rencontre avec les pays orientaux a représenté pour Lamartine, par exemple, un

processus de remémoration. Pour une part, c'est vivre les histoires légendaires ou authentiques qui ont bâti sa formation; pour une autre part, c'est même quelquefois abolir la durée pour contempler lors du voyage ce qui a fait l'Histoire de l'Autre; c'est un lieu commun pour le voyageur du XIX ${ }^{\text {ème }}$ siècle de retrouver en Orient l'époque médiévale duquel il rêvait. Le voyageur peut feuilleter à loisir les pages de sa propre histoire en parcourant les terres des civilisations bien disparues depuis de longs siècles. 
La partie la plus vaste du mouvement d'orientalissme est donc le voyage, d'où l'époque de François I, la France jouissait d'une bonne relation avec la Turquie ce qui a donné la chance aux ambassadeurs de résider pour longtemps à Constantinople. Cette résidence confortable leur a permis d'accompagner un nombre d'écrivainsvoyageurs, soit des historiens, des hommes de lettres ou des artistes, et leur fournissent par toutes les informations qui leur facilitent l'écriture de leur récit de voyage. Il s'agit donc d'une description des lieux, des coutumes et traditions, de la culture et du peuple. D'où, le récit de voyage a joué un rôle principal et fondamental dans cette présentation de l'Autre et a donné le lecteur cette possibilité de bien le reconnaitre de loin. Le désir d'écrire sur cet Autre est pour les écrivains du XIX ${ }^{\text {ème }}$ siècle une part d'eux-mêmes. Il est devenu de plus en plus un goût qui dominait les mouvements littéraire et artistique. Ce goût a dominé leur inspiration et a capté leurs œuvres. À travers de ces récits, ils cherchaient la réponse au questionnement des origines. Le voyage en Orient, est donc le retour aux sources de l'humanité et aux débuts de l'Histoire. D'Égypte à Jérusalem, les écrivains-voyageurs se sont fixés des étapes initiatiques pour accéder au paradis perdu de ce monde mythique. Il s'agit de retrouver dans l'Orient l'authenticité perdue et dans les lieux mythiques le berceau de la civilisation occidentale. Désireux de renouveler leurs modèles d'écriture et leur inspiration, spécialement après la révolution industrielle, les écrivains du XIX ${ }^{\text {ème }}$ siècle ont été séduits par la puissance du dépaysement en Orient dans lequel ils puisaient avant tout des thèmes nouveaux: la cruauté du tyran (comme le personnage de Pharaon au Roman de la momie de Gautier), le désert et la sensualité. Ils y ont trouvé aussi l'occasion de décrire, avec la véracité des écrits des orientalistes et leurs images éclatantes, des aspects des civilisations bien disparues depuis des siècles.

Le voyage est donc ce moyen magnifique qui a mené d'entrer en contact avec l'Autre et a constitué un procédé de confrontation avec un univers inhabituel. Il s'agit alors d'un voyage réel que l'homme fait par le corps et la pensée et un voyage imaginaire que fait l'homme seulement par la pensée. Dans le voyage réel, le corps se met d'un endroit à l'autre dont l'esprit s'engage avec lui. Le voyageur, le penseur, l'écrivain ou le peintre plonge physiquement et mentalement dans ce monde inconnu et étranger. Le goût de voyage donne encore une chance de reconnaître, de rencontrer et de faire 
une expérience de l'Autre inconnu. Au contraire, le voyage imaginaire engage seulement l'esprit, c'est-à-dire qu'il donne beaucoup de détails pleins des aspects concernant le rêveur: ses sentiments, ses pensées et ses angoisses sur l'autre:

«Le voyage imaginaire est donc une sorte de déplacement sans mouvement. Il peut être une étape préalable avant d'effectuer un véritable voyage. C'est un parcours songé qu'on effectue à l'intérieur de soi-même et qui n'exige pas de traverser un chemin.» (Abdelkader 2008: P. 2)

Par ailleurs, le voyage se fait de deux façons: dans l'espace et dans le temps. Le premier oblige le rêveur de se déplacer pour découvrir un monde plein des détails utiles, comme les paysages, la culture, la civilisation et les traditions. Mais, le voyage dans le temps approfondit seulement l'étude de l'histoire et la recherche des origines. De plus, ce voyageur sera incité de découvrir les civilisations disparues; c'est le cas par exemple des visites d'un nombre des écrivains-voyageurs des vestiges de la civilisation pharaonique. De ce fait, le voyage dans le temps représente une étape alternative pour le voyage spatial. Un nombre de voyageurs sont partis pour faire des rencontres avec l'Autre afin d'avoir des contacts avec une autre culture, une autre civilisation et découvrir d'autres mœurs. Le privilège du voyage réel est que le voyageur a la chance de noter ses remarques et ses souvenirs personnels dans un récit de voyage autour du pays et du peuple visités:

«Lors de son voyage, l'écrivain peut rédiger un journal de bord, noter ses souvenirs personnels dans un carnet et écrire. des lettres adressées à ses proches ou à ses amis. Ainsi, le récit de voyage repose sur une écriture du déplacement. De ce fait, le voyage et l'écriture représentent deux activités complémentaires. » (Ibid.: 3 )

L'Autre et son image ont occupé conséquemment une place centrale dans les récits de voyage, qui sont les premières références pour plusieurs textes historiques et littéraires au XIX ${ }^{\text {ème }}$ siècle. Le Voyage en Orient de Gérard de Nerval a été pour longtemps comme un guide utile avec un rôle fort significatif pour un nombre des voyageurs et des hommes de lettre. Dans son œuvre, il a enregistré des informations riches et multiples sur la vie sociale en Égypte, la religion d'Islam et les mythes des 
pharaons. Il n'a pas voyagé pour visiter la Haute-Égypte, et a préféré de rester au Caire pour contempler les aspects de la vie quotidienne dans cette ville:

« Nerval est l'un des seuls voyageurs français à avoir présenté à son lecteur les traditions des Égyptiens: il a expliqué leurs croyances et a fait preuve de curiosité envers leurs religions, aussi bien anciennes que mystérieuses Son attitude vis-à-vis de l'Autre (les Égyptiens) est-complètement différente de ses prédécesseurs. » (Ibid.: 6)

C'est au XVII ${ }^{\text {ème }}$ siècle que l'orientalisme s'est inscrit comme une des pensées humanistes de la Renaissance, où il y avait un grand intérêt au christianisme oriental. Par conséquence, il était indispensable la traduction d'un nombre des textes écrits en arabe ou en syriaque et qualifier un nombre des interprètes qui perfectionnent un nombre des langues orientales: arabe, persan, hébreu ou syriaque. La traduction des Mille et une nuits, grâce à Antoine Galland en 1704, a piqué la curiosité du lecteur occidental qui sentait un nouvel charme sensuel et unique en découvrant des histoires totalement étrangères et des mythes très anciens. De celles traductions ont engendré un nouvel goût sans précédent aux milieux occidentaux. En 1721, Montesquieu a publié ses Lettres persanes dont l'axe était l'Autre oriental dans un roman épistolaire. Cette œuvre a exprimé, à travers les idées philosophiques de son auteur, l'altérité, les relations et les échanges entre les deux civilisations: occidentale et orientale. En 1747, Voltaire a écrit Zadig dont les événements se déroulaient dans un royaume imaginaire quelque part entre l'Égypte et Babylone. Les œuvres se sont variées et se considéraient comme des miroirs de cette civilisation bien disparue depuis de longs siècles. Et sans doute, ces œuvres ont guidé l'Europe dans sa recherche de reconnaître l'Égypte ancienne et les mythes pharaoniques:

«La civilisation égyptienne est la plus ancienne civilisation au monde (la civilisation babylonienne lui est peut-être légèrement antérieure). Nous n'en connaîtrons jamais d'autres aussi intimement car la particularité du climat égyptien a permis la conservation non seulement des différents monuments mais aussi des écrits, des objets usuels et même des corps (momies).» (Young 1995: 12) 
Après l'expédition de Bonaparte en Égypte entre 1798 et 1801, les recherches se sont poursuivies en étudiant la vie pendant l'époque des familles pharaoniques, les momies et la momification (un procédé encore secret jusqu'à maintenant), l'écriture hiéroglyphique (déchiffrée par Champollion) et aussi la prédominance de l'époque pharaonique dans les différents domaines de la science: la médecine, la physique, la chimie et l'architecture.

\subsection{Secret et importance de l'intérêt pour l'Égypte}

Le voyage en Orient a fait partie de ce vaste mouvement de curiosité amorcé au XVIII ${ }^{\text {ème }}$ siècle et qui s'était emparé de l'Europe. Bien peu d'artistes se déplaçaient réellement avant la fin du siècle et les contacts se firent d'abord par l'intermédiaire des ambassades. La France jouissait d'un statut privilégié avec la Turquie depuis François Ier. Cette alliance franco-turque autorisait les ambassadeurs à résider de manière permanente à Constantinople. Cette position confortable leur permettait d'accueillir les artistes voyageurs et de leur délivrer des autorisations pour faciliter leur déplacement dans la ville, lumière de l'Occident.

Un grand nombre d'écrivain est parti avec l'espoir de découvrir l'autre face du monde avec un enthousiasme fort pour déceler les mythes orientaux. Ils sont revenus pour enrichir la production littéraire et artistique avec des ouvrages majeurs. Toutefois, en marge de cette littérature savante et de la culture officielle, les ouvrages publiés par les voyageurs connurent un intérêt et un succès sans précédent aux yeux d'une nouvelle clientèle privée. C'est essentiellement par l'édition et les récits de voyage que les thèmes orientalistes se sont propagés. Dans le XVIII ${ }^{\text {ème }}$ siècle, les œuvres de vulgarisation se multiplièrent sans grande fiabilité car les auteurs n'hésitaient pas à se copier les uns des autres, à extrapoler d'une manière assez fantastique. Aux XVII ${ }^{\text {ème }}$ et XVIII ${ }^{\text {ème }}$ siècles, seuls quelques rares voyageurs osaient s'aventurer audelà de la Méditerranée et les aristocrates britanniques s'adonnaient à ce que l'on appelait Le Grand Tour qui, à la fin des années 1730, repoussait les frontières de l'Europe toujours plus à l'Est, en Asie Mineure et en Égypte. Mais la question qui se pose ici est: pourquoi les orientalistes s'intéressaient à ce point par à la civilisation d'Égypte antique? 
"Pays venu des profondeurs de l'histoire, l'Égypte exerce beaucoup de fascination chez les orientalistes, les épris d'archéologie ou tout simplement les touristes en quête de dépaysement ou de soleil.» (Blanc 2010: 13)

Nous pouvons reprocher à ces voyageurs, ces orientalistes et ces missions exploratrices qui n'avaient d'autre but que la découverte des secrets d'une antiquité assez disparue depuis des siècles. Nous sommes en droit de s'interroger sur ce manque d'intérêt pour une culture et une science supérieures ayant produit des savants tels qu'Ibn Sina, père de la médecine - pourtant bien connu en Europe sous le nom d'Avicenne - et Jabir Ibn Hayyan, - latinisé en Geber au moyen âge- père de la chimie, dont les œuvres sont répandues dans tous les pays arabes. L'Égypte a été le théâtre de plusieurs occupations successives: le contrôle Ottomane, l'occupation française qui reste trois ans en Égypte suite à l'expédition de Napoléon Bonaparte de 1798 à 1801, et enfin l'occupation anglaise qui a dominé sur le pouvoir en Égypte après le départ de l'armée française et y a demeure environs de 60 ans. Avec chaque occupation, l'occupant faisait tout son possible pour imposer son empreinte. Il ne faut pas oublier le rêve pharaonique du jeune Bonaparte, ce général qui poursuivait le rêve de monter sur le trône d'Égypte, sachant bien que celui qui possède l'Égypte peut posséder le monde. Il prétendait apporter les lumières de la Révolution française aux Égyptiens:

«Avec les Armées, la langue française fit son entrée en Égypte. Or, l'expédition d'Abu Naparti n'était pas seulement une expédition militaire: une foule de scientifiques (Vivant Denon, Conté, Monge...) débarqua avec les canons. Il en résulta la célèbre Description de l'Égypte, et l'Égypte devint une passion française. De plus, les Français créèrent l'Institut d'Égypte, et ouvrirent une bibliothèque. » (Goes 2004: 37)

D'une part, c'est avec l'Égypte qu'il entendait réaliser l'utopie d'un empire français, et d'autre part, les européens ont accédé la civilisation arabe à travers Andalousie.

\section{L'Égypte comme une source d'inspiration}


Les récits des écrivains voyageurs ont joué un grand rôle dans la représentation de la civilisation orientale avec ses traditions, ses habitudes, ses paysages, ses populations et son histoire dans la production littéraire et artistique occidentales. Les écrivainsvoyageurs du XIX ${ }^{\text {ème }}$ siècle avaient le but d'analyser l'image des pays orientaux, prenant comme exemple, Gérard de Nerval, qui a visité le Caire et a réussi de décrire la vie contemporaine dans la ville capitale. L'analyse des œuvres des Orientalistes et des écrivains-voyageurs montre que l'antiquité égyptienne représente l'une des plus importantes sources d'inspiration dans la littérature française tout au long de son histoire. Dans ces œuvres, qui ont inondé le XVI ${ }^{\text {ème }}$ siècle et se suivaient jusqu'au $\mathrm{XIX}^{\text {ème }}$ siècle, les écrivains ont mis l'accent sur la relation de double nature qui relie entre l'Orient et l'Occident:

«Dans la seconde moitié du XIXème siècle, les Français envoient des techniciens en Égypte, désireuse de se détacher de l'Empire ottoman; parallèlement, les Français et les Anglais exercent une tutelle sur les finances de la Porte. F. de Lesseps fait creuser le canal de Suez (1859-1869), à une époque où l'Égypte est devenue semi indépendante des Turcs (règne de Sä̈d Pacha, fils de Méhémet Pacha). Très endettée, elle s'est tournée vers la France et l'Empereur Napoléon III. » (Ibid.: 38)

Tout au long du XIX ${ }^{\text {ème }}$ siècle, de nombreux écrivains ont puisé à la source des histoires d'Égypte antique, et certains ont écrit sur les mythes pharaoniques, comme par exemple, Une nuit de Cléopâtre de Théophile Gautier. La production littéraire de ce siècle était très riche. Aussi reste-t-il pour la littérature française un âge d'or, avec de très nombreux chef d'œuvre qui ont laissé percevoir, au-delà des courants littéraires qui se succédaient, des créateurs aux fortes personnalités artistiques. Mais la présence de l'antiquité égyptienne dans le mouvement artistique et littéraire et la passion française ne cachait pas celle de la France en Orient:

«Traditionnellement existaient au Proche-Orient arabe plusieurs foyers de haute culture française: au Caire, l'Institut français d'archéologie orientale (IFAO), à Damas, l'Institut français d'études arabes et à Beyrouth, l'Institut de recherche sur l'archéologie et l'histoire du Proche-Orient. .... N'oublions pas non plus qu'après Auguste Mariette des Français se sont succédé à la tête 
du grand musée égyptien du Caire jusqu'à la période de Nasser. Jusqu'à nos jours, l'Égypte reste une grande passion française.» (Goes 2004: P. 39)

Les centres français ont été répandus en Orient: au Caire, à Beyrouth et à Bagdad et accueillaient des gens de tout âge. Il y avait aussi des institutions éducatives et religieuses qui représentaient la présence française en Orient:

«Des centres culturels existent à Bagdad (jusqu'à la première crise du Golfe), en Jordanie (Amman), et encore au Liban (Beyrouth, Saïda, Zahlé et Tripoli). Ils accueillent des élèves de tous âges.» (Ibid.: 40)

On peut conséquemment parler d'une littérature libanaise ou égyptienne rédigée en Français, mais il reste encore un peu limité cette forme de production littéraire dans les autres pays orientaux et arabes. La littérature française du XIX ${ }^{\text {ème }}$ siècle se définit par deux dates repères: 1798, date du coup d'État de Bonaparte qui a instauré le Consulat, et a mis fin à la période révolutionnaire, et 1899, moment de résolution des tensions de l'affaire Dreyfus et de la menace du Boulangisme, cette date à laquelle se sont imposées finalement les valeurs de la IIIe République. La modernité littéraire s'est affirmée dans ce siècle avec des courants marquants qui touchaient tous les arts, comme le Romantisme, le Réalisme, le Naturalisme ou le Symbolisme.

$\mathrm{Au}$ cours du $\mathrm{XIX}^{\text {ème }}$ siècle, les créateurs ont offert des œuvres multiples, particulièrement dans le domaine de la poésie avec (Lamartine, Vigny, Musset, Hugo et Baudelaire) tout comme dans le domaine du roman avec (Stendhal, Balzac, Dumas, Hugo, Flaubert et Gautier) et dans une moindre mesure au théâtre avec (Musset, Hugo et Edmond Rostand). Le roman était le genre dominant par l'intérêt qu'il a suscité, par sa diffusion massive entretenue par l'instruction publique croissante et le développement de la presse et des feuilletons dans la deuxième moitié du siècle. La plupart des romanciers ont été issus de la bourgeoisie et ont vécus désormais de leur plume. La multiplication des formes romanesques était une marque de cette époque. Le roman du moi: voisin de l'autobiographie qu'illustre les Mémoires d'outre-tombe de Chateaubriand (1848), le roman autobiographique à la première personne marquait le début du siècle avec le goût pour la confession intime cachée derrière un prêtenom, en associant lyrisme et narcissisme pour explorer le mal de vivre d'une génération. Il constituait l'un des résultats importants du romantisme dans la 
littérature du $\mathrm{XIX}^{\text {ème }}$ siècle avec des œuvres personnelles comme René (Chateaubriand -1802), Corinne (Madame de Staël -1807), Adolphe (Benjamin Constant -1816) ou La Confession d'un enfant du siècle (Musset -1836). Le roman historique: sous l'influence de Walter Scott, les auteurs cultivaient la nostalgie et le pittoresque avec un souci de documentation et de recréation du passé en mêlant personnages et faits imaginés à des personnages et des actions historiques:

«D'entrée de jeu, signalons que généralement les romans historiques n'utilisent le plus souvent l'histoire que comme cadre de récit.» (Pouliot 1996:

6)

En Angleterre, avec le premier roman historique pour la jeunesse: Les enfants de la Nouvelle Forêt (1847) de F. Marryat, l'auteur a fait revivre l'époque de la guerre civile. Il y avait aussi quelques titres exemplaires: Cinq-Mars de Vigny - 1828, Notre-Dame de Paris de Victor Hugo - 1831, Les Trois Mousquetaires Alexandre Dumas père - 1844, Le Bossu de Paul Féval - 1858. Le genre s'est prolongé tout au long du siècle avec quelques œuvres saillantes comme le Roman de la momie de Gautier - 1857 et Salammbô de Flaubert - 1862.

Les principales caractéristiques des romans historiques contemporains sont de se situer dans la continuité d'une tradition déjà ancienne d'une part et, d'autre part, de se dérouler désormais à des époques encore plus lointaines que celle de la France médiévale, ou de l'Égypte antique. En dépit d'un qualificatif commun, il y aurait en quelque sorte deux types de roman historique: celui qui se rattache à la tradition scottienne (chez Walter Scott, le récit est axé sur un personnage fictif); et celui qu'il est convenu d'appeler roman panoramique puisqu'il met en scène de nombreux personnages dont aucun n'est central. Dans le XIX ${ }^{\mathrm{ème}}$ siècle, le roman réaliste a paru, dont il s'agit de créer un certain effet réel en peignant avec un souci constant du détail et de la vraisemblance les décors, les personnages et les faits. Les expressions de Stendhal (roman = miroir) ou de Balzac (romancier $=$ historien du présent) ont montré dans la première moitié du siècle une voie que Flaubert et Maupassant ont approfondi. En conséquence, la production littéraire du XIX ème siècle a fait parallèlement une large place au roman d'apprentissage, en accompagnant les débuts dans la vie sociale des personnages. De nombreux romanciers ont participé aussi à la richesse de la 
création romanesque réaliste dans la seconde moitié du siècle: tel que les frères Goncourt (Edmond et Jules) avec leur minutie descriptive (Germinie Lacerteux 1865), Jules Verne qui a inauguré ses romans d'aventure et d'anticipation avec Cinq semaines en 1863 ou Alphonse Daudet (Le Petit Chose - 1868) et Jules Vallès ( L'Enfant -1879) qui s'inspirent de leur propre vie. La fin du XIX ${ }^{\text {ème }}$ siècle a été marquée par une réaction contre le courant du réalisme et par une attitude idéaliste associée à un retour à la perspective religieuse avec Huysmans (À Rebours en 1884), Léon Bloy (Le Désespéré en 1886) ou Maurice Barrès (Les Déracinés en 1897). D'autres mouvements ont paru comme l'exotisme: Pierre Loti (Pêcheur d'Islande en 1886) et le siècle a témoigné la naissance du roman d'analyse "pré proustien" avec Paul Bourget (Le Disciple en 1889) ou Anatole France (Le Lys Rouge en 1894).

Donc, le roman n'avait pas de nature spécifique, spécialement au début du XIX ${ }^{\text {ème }}$ siècle. Ce siècle témoignait une politique particulièrement instable. Huit régimes se sont succédés entre 1800 et 1900, et les écrivains ont représenté une partie de la vie intellectuelle quel que soit leur personnalité littéraire ou leur direction politique. Les intellectuels, héritiers du siècle des Lumières et de la Révolution française, ont exprimé une forte confiance pour établir une société meilleure, malheureusement avant 1840, le genre romanesque était en marge. Conséquemment, les auteurs se dirigeaient vers d'autres formes comme: les mémoires et les poèmes en prose. Mais au début des années quarante, les mythes pharaoniques ont envahi la production romanesque du siècle. Après 1848 , le roman a témoigné son âge d'or, et la puissance du roman réaliste est parue. De nombreux romanciers ont participé aussi à la richesse de la création romanesque réaliste dans la seconde moitié du siècle.

Les histoires des rois et des reines pharaoniques incitaient certains concepts, comme celui de la civilisation, du tyran et de la prospérité. D’autres hommes de Lettres écrivaient sur les mythes pharaoniques. Ces mythes contiennent quelques fois des détails imaginaires et peu crédibles comme la momie qui est revenue d'entre les morts, chassant les gens et éveillant l'horreur et la peur dans leurs âmes, et elle est venue se venger de celui qui s'est approché de son tombeau. Ce genre des textes est également considéré comme une matière très riche pour les romans et les films d'horreur spécialement du $X^{\text {ème }}$ siècle. Avec le début de ce siècle, les textes 
européens qui racontent des histoires sur les momies égyptiennes qui revivent après la mort foisonnent, Gautier, Nerval, Lamartine et Chateaubriand ont enrichi ce foisonnement. Enfin, si les histoires des mythes pharaoniques ont incité les concepts de la civilisation et du tyran, les histoires fictives des momies ont suscité la peur et l'horreur, ces dernières représentaient une source vierge d'inspiration pour les auteurs d'histoires d'horreur.

\section{Conclusion}

L'expédition de Napoléon Bonaparte sur le pays du Nil en 1798 et jusqu'à 1801 avait fortifié le goût pour une antiquité bien disparue depuis de longs siècles. Cette fascination a pu enrichir la littérature et l'art français et a aidé à faire naître un phénomène appelé l'égyptomanie. Le goût pour l'Égypte, le voyage en Orient et le désir de reconnaître l'Autre ont capté la pensée des hommes de lettres. D'une part, l'imaginaire a envahi la production littéraire, grâce aux mythes pharaoniques et des mystères de l'antiquité la plus ancienne du monde, et d'autre part, il a été né une certaine nécessité de référer aux œuvres des orientalistes et des écrivains voyageurs pour garantir l'authenticité du texte. Le courant d'orientalisme a commencé à être connu grâce aux efforts des missionnaires au Moyen âge. Ils voyageaient pour l'Orient avec le but d'apprendre ses langues et découvrir ses religions. Au Moyen Âge, ces missionnaires partaient avec des motifs de piété, le désir de voir et de s'instruire et des raisons politiques conduisaient les voyageurs en Orient. Les voyageurs du XVI ${ }^{\mathrm{ème}}$ siècle avaient la curiosité des hommes de la Renaissance. Ils promènent le lecteur dans les lieux qu'ils ont visités et lui communiquent leurs impressions et leurs expériences. Ils ont ouvert la voie aux chercheurs de l'âge suivant. L'orientalisme incarne alors cette fascination et cet intérêt occidentaux pour l'Orient. Des écrivains-voyageurs comme Lamartine, Nerval et Gautier ont encouragé ce mouvement en transmettant leurs expériences et leurs impressions dans des récits littéraires et récits de voyage.

La recherche de l'Autre est une façon de satisfaire le désir d'évasion, de sortir de soimême. Ce goût se retrouve dans les récits historiques comme Notre-Dame de Paris, les récits de voyage España de Gautier ou les romans dont l'action se déroule dans des lieux et temps lointains comme le Roman de la Momie. Alors, l'Égypte a exercé 
une fascination particulière sur l'imaginaire romantique en raison de son statut de berceau de toute civilisation et de son obsession de l'immortalité et de ses énigmes qui excitent les chercheurs et les hommes de Lettres, comme par exemple, l'écriture hiéroglyphique:

"Le système d'écriture des anciens Égyptiens ne cesse d'inspirer les écrivains au fil du siècle et ce, bien souvent, dans une plus ou moins grande ignorance de la langue dont l'écriture était le support. Maintes pages de fiction en prose, des poèmes et des réflexions en attestent, publiés par Honoré de Balzac, Victor Hugo, Gérard de Nerval, Théophile Gautier, Charles Baudelaire, Gustave Flaubert, Villiers de L'Isle Adam, José Maria de Hérédia, Stéphane Mallarmé ou encore Paul Valéry. Près d'une cinquantaine d'ouvrages et plusieurs centaines d'articles et de parties de volumes relevant de l'«érudition» (exotérique et ésotérique) s'ajoutent au corpus de ces écrivains de vocation.» (Lançon 2011: 1)

L’Égypte pharaonique avait toujours fasciné les Occidentaux. Cette civilisation la plus ancienne au cours de toute l'humanité a affecté d'une manière ou d'autre les aspects sociaux, scientifiques et littéraires de la civilisation occidentale. Avec ses quatre mille ans d'histoire, l'Égypte antique a eu beaucoup de traces que n'importe quelle autre civilisation. L'unicité de sa gloire, de son histoire, de sa culture et de son art a depuis le siècle des romains fasciné les voyageurs, les savants et les archéologues. Ses trésors ont été, et restent jusqu'à maintenant, sur la tête des listes de la recherche scientifique, littéraire et historique. La diffusion de ce grand nombre de monuments et d'antiquités égyptiens dans la plupart des musées mondiaux permet aux autres peuples et aux autres civilisations de connaître et de reconnaitre du près tous les aspects de la vie pharaonique. À défaut d'être véritablement connue, l'Égypte est présente dans la conscience et dans l'imaginaire des européens. Mais, c'est au XIX $^{\text {ème }}$ siècle, à la suite de l'expédition de Bonaparte (1798-1801), qu'elle est devenue l'objet d'une véritable fascination.

\section{Bibliographie}


ABDelKaDer, Hamdi Abdelazim Abdelmaksoud. 2008. L'Égypte dans Voyage en Orient de Gérard de Nerval et la France dans L'or de Paris de Rifaa Al Tahtawi, Université du Québec à Montréal

AFFAYA, Mohammed Nour Eddine. 1999. La communication de l'interculturel entre le réel et le virtuel, Afers Internacionals, Espagne, Nr. 43-44, P. 217-241.

Blanc, Pierre. 2010. Égypte, une géopolitique de la fragilité, Confluences méditerranée, Nr. 75, P. 13-31.

ALSAID, Mouna. 2009. L'image de l'Orient chez quelques écrivains français: Lamartine, Nerval, Barrès et Benoit. Naissance, évolution et déclin d'un mythe orientaliste de 1'ère coloniale. Université Lumière. Lyon 2. École doctorale: Lettres, langues, linguistique, arts.

APOSTOLOU, Irini. 2009. L'orientalisme des voyageurs français au XVIII ème siècle. Une iconographie de l'Orient méditerranéen. Presse de l'université Paris Sorbonne CHAOUACHI, Salaheddin. 2005. Les sensations orientales et le merveilleux dans l'œuvre de Théophile Gautier. Faculté des sciences humaines et sociales. Centre de publication universitaire. Tunisie.

FOLEY, François. 2008. Eil d'Horus et Calame d'Thot. Mesure et représentation de L'Égypte pharaonique dans la littérature française du XIX siècle. Un doctorat en études littéraires. Université du Québec à Montréal

Gautier, Théophile. 1966. le Roman de la momie. Garnier - Flammarion. Paris.

GAUTIER, Théophile. 1996. Voyage en Égypte. La Boîte à documents. Paris.

GoES, Jan. 2004. Littératures francophones du monde arabe. La France et le Machreq (Proche-Orient). Overgenomen uit Romaniac. Nr. 95. P. 34-44

HoRnUNG, Erik. 1996. L'esprit du temps des pharaons. Traduit d'allemand par Michèle Hulin, Philippe Lebaud. Éditions du Félin, Paris,

LAFOUGE, Jean Pierre. 1988. Étude sur l'orientalisme d'Eugène Fromentin dans ses «Récits algériens». Peter Lang publishing. New York

Lançon, Daniel. 2011. L'imaginaire des hiéroglyphes chez les écrivains français au XIXe siècle: égyptosophie, cratylisme et analyse de la psyché. Université Grénoble. Traverses ET 19-21. P. 1-15 
LAURENS, Henry. 1987. Les origines intellectuelles de l'expédition d'Égypte: l'orientalisme islamisant en France; (1698 - 1798). Istanbul.

LAURENS, Henry. 1989. L'expédition en Égypte, Paris, Armand Colin.

LAURENS, Henry. 2000. L'Orient arabe. Arabisme et islamisme de 1798 à 1945. Armand Colin. Paris.

MOURA, Jean-Marc. 1998. La littérature des lointains. Histoire de l'exotisme européen au XXe siècle. Champion. Paris.

Moussa, Sarga. 2000. Alphonse de Lamartine. Voyage en Orient. Éditions Champion. Paris.

NoDIER, Charles. 1989. Du fantastique en littérature. SL. Chimères. Coll. « Barbe bleue ». France

Peltre, Christine. 2003. Les orientalistes. Nouvelle édition corrigée. Édition Hazan.

Pouliot, Suzanne. 1996. Le roman historique: lieu idéologique et identitaire. Lurelu. Vol. 18. NR. 3. P. 6-11

SAÏD, Edward, 2000. Culture et impérialisme. Traduit de l'anglais par Paul Chemla. Librairie Arthème Fayard. Le monde diplomatique.

SAÏD, Edward. 2005. L'Orientalisme: L'Orient créé par l'Occident. Traduit de l'américain par Catherine Malamoud. Éditions du Seuil. Paris.

TOHME JARrouche, Lisette. 1997. Descriptions et descriptif dans la prose romanesque de Théophile Gautier. Dissertation. Université de Paris.

YounG, J. C. 1995. Le colonialisme et la machine désirante. Traduit par Isabelle Lee. Routledge. London. 